\title{
Unstaged Diffuse Large B-Cell Lymphoma in the United States: Predictors and Patient Outcomes
}

\author{
AMIR BISTA $^{1}$, SANDHYA SHARMA ${ }^{1}$, TAUFIQ RIJWANI ${ }^{2}$ and BINAY K. SHAH ${ }^{2,3}$ \\ ${ }^{1}$ Guthrie Robert Packer Hospital, Sayre, PA, U.S.A.; \\ ${ }^{2}$ Pacific Northwest University of Health Sciences, Yakima, WA, U.S.A.; \\ ${ }^{3}$ Northwest Puget Cancer Center, Sedro-Woolley, WA, U.S.A.
}

\begin{abstract}
Background: Treatment and prognosis of diffuse large B-cell lymphoma (DLBCL) depends on the stage of lymphoma. We conducted this study to examine unstaged DLBCL in the United States. Materials and Methods: We used Surveillance Epidemiology and End Result (SEER) 18 registries to select patients with DLBCL diagnosed during January 2000 to December 2012. Limited regional distant Summary stage 2000 was used to determine stage of the disease as localized, regional, distant or unstaged. We used logistic regression to investigate factors associated with unstaged DLBCL. Cox proportional hazards model was used to compare survival outcomes. Results: Among 67,765 patients, disease in 3,194 (4.71\%) was unstaged. Age $(60+y e a r s)$, non-African American, not married marital status, metropolitan residence, median household income $>\$ 50,000$, lymph node as the primary site and those with other primary malignancies before diagnosis of DLBCL were the factors associated with cases being unstaged. The 5-year relative survival rate for patients with unstaged DLBCL was inferior to that of those with localized and regional disease, and superior to that of those with distant disease (hazard ratios of 0.58, 0.66 and 1.24 for localized, regional and distant disease, respectively, when compared to unstaged cases). Conclusion: Several factors are associated with higher risk of unstaged DLBCL. Patients with unstaged $D L B C$ had significantly inferior survival rates compared to patients with localized and regional stage.

Non-Hodgkin lymphoma (NHL) is a group of hematological
\end{abstract}

This study was presented as a poster at the American Society of Hematology Annual Meeting and Exposition (December 5-8, 2015) in Orlando, FL.

Correspondence to: Dr. Binay Kumar Shah, North Puget Cancer Center, 2000 Hospital Drive, Sedro-Woolley, WA 98284, U.S.A. Tel: +1 3608566021, Fax: +1 360856752, e-mail: binay.shah@gmail.com

Key Words: Lymphoma, staging, SEER, survival. malignancies, occurring at an annual incidence of approximately 20 per 100,000 (1). Diffuse large B-cell lymphoma (DLBCL) is the most common type of B-cell NHL, comprising approximately $30 \%$ of all cases (2). It is an aggressive cancer that can be cured in over $50 \%$ of all patients (3). Staging lymphoma helps define disease location and extent, and also provides a baseline along which treatment response and disease progression can be monitored. In addition, staging helps determine the therapeutic plan for the patient.

However, each year a small percentage of newly diagnosed cancers are not assigned a tumor stage. Merrill et al. reviewed the Surveillance, Epidemiology and End Results (SEER) database in order to further characterize the extent of unstaged solid tumor malignancies. They found a higher incidence of unstaged disease in 10 out of 18 cancer types in those of African-American race, in elderly individuals, and in the uninsured (4). Importantly, they reported an inferior 5 -year survival in patients with unstaged disease. Similarly, Worthington et al. reviewed the SEER database and reported that tumor in $5.1 \%$ of patients with colon cancer and $7.8 \%$ of those with rectal cancer were unstaged, with characteristics such as older age, African-American race and female sex being associated with a higher likelihood of having unstaged disease (5).

We conducted this study to examine unstaged DLBCL, taking into account both patient characteristics and disease prognosis.

\section{Materials and Methods}

Data. SEER is a large population-based database that collects cancer statistics from various sources around the country. The SEER 18 registries were reviewed to select patients diagnosed with DLBCL between January 2000 and December 2012. These 18 registries comprise approximately $27.8 \%$ of the US population, and include data from Atlanta, Connecticut, Detroit, Hawaii, Iowa, New Mexico, San Francisco-Oakland, Seattle-Puget Sound, Utah, Los Angeles, San Jose-Monterey, Rural Georgia, Alaska Native Tumor, Greater California, Kentucky, Louisiana, New Jersey, and Greater 


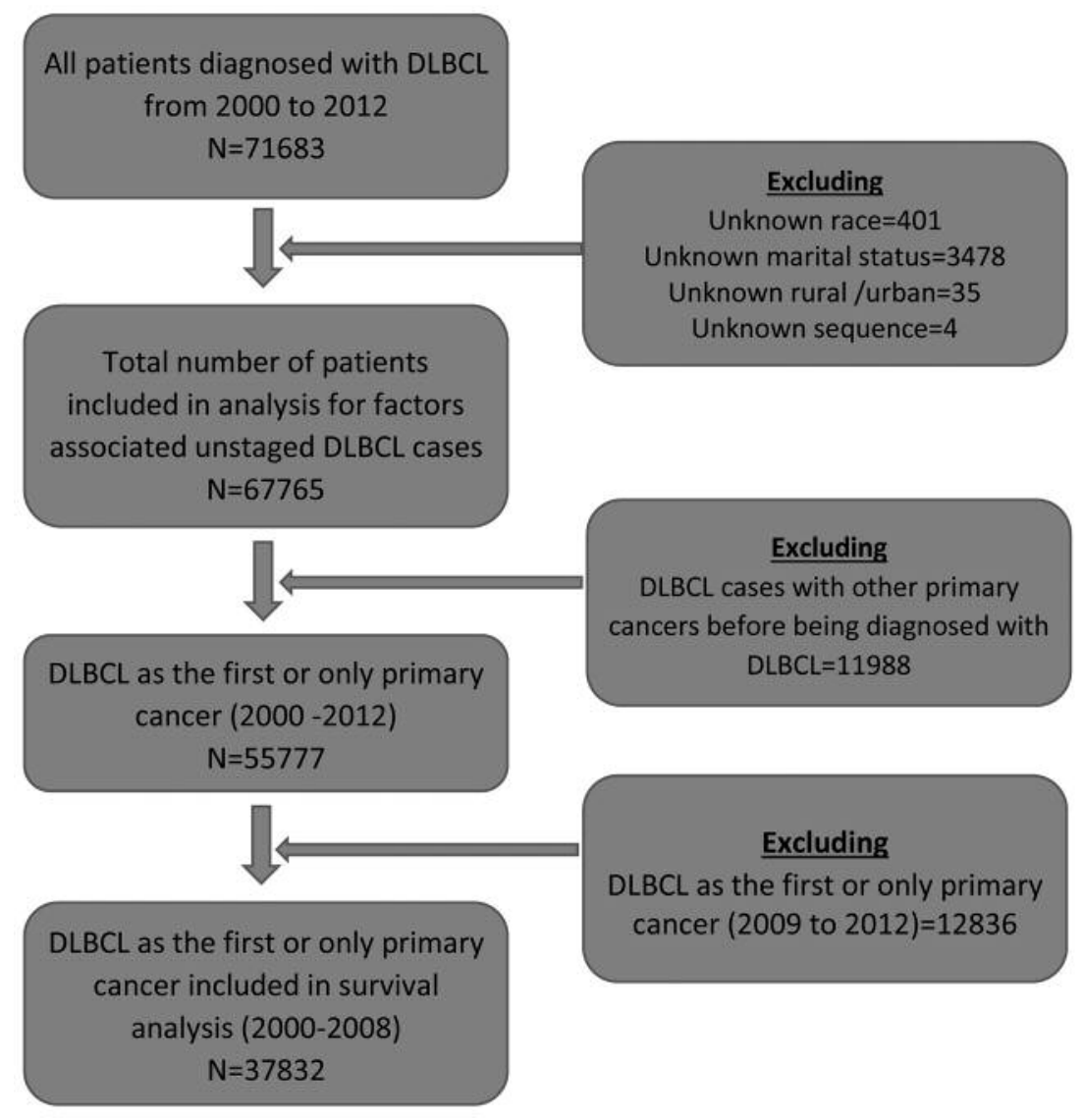

Figure 1. CONSORT diagram for patients with diffuse large B-cell lymphoma (DLBCL).

Georgia (6). These records were sorted, and patients with unknown race, marital status, area of residence or sequence of cancer, were excluded from our study (Figure 1).

Data variables. The patients of the study were divided into various subgroups based on age (0-59 years or 60 years and older), sex (male or female), race (Caucasian, African American, or other\}, marital status (married, single or separated/divorced/widowed), place of residence (rural, urban or metropolitan), annual household income $(\$ 0-25,000, \$ 25,000-50,000$, or $\$ 50,000$ and higher), sequence of cancer (primary DLBCL or other primary malignancy prior to diagnosis of DLBCL) and site of primary malignancy (lymph node, extralymphatic, or unknown site of primary).

Statistical techniques. Local, regional, and distant (LRD) summary staging was used to classify cases of DLBCL. Patient records that did not contain any staging information were categorized as unstaged disease. Factors associated with unstaged cancer were then analyzed using a binomial logistic regression analysis to compute unadjusted (uOR) and adjusted odds ratios (aOR). Corresponding two-sided p-values were calculated, with $p<0.05$ considered statistically significant.

Relative 3- and 5-year survival (RS) ratios were calculated for the various cohorts based on age, sex, and race, using SEER*Stat software version 8.2.1, National Cancer Institue (Bethesda, MD,
USA) and compared using a z-score and corresponding two-sided $p$-values. Relative survival is the ratio of the proportion of observed survivors in a cohort of patients with cancer to the proportion of expected survivors in a comparable set of cancerfree individuals within a specific time period. Cox proportion hazards model was used to compare the survival outcomes for different stages of DLBCL, after adjusting for age, sex, and race. Survival curves were then created based on actuarial tables for different cancer stages.

\section{Results}

Of the 67,765 patients with DLBCL that were included in the study, 3,194 (4.71\%) had unstaged disease. Demographics of patients are included in Table I. Unstaged cases were more common among individuals above the age of 60 years (uOR=1.478, $p<0.001 ; \mathrm{aOR}=1.458, p<0.001)$. After adjusting for variables, males and females were equally likely to be unstaged. Compared to Caucasians, disease in African Americans was less likely to be unstaged ( $\mathrm{uOR}=0.804$, $p<0.005 ; \mathrm{aOR}=0.835, p<0.022$ ), whereas disease in 'Other' races was more likely to be unstaged after adjusting for other variables $(\mathrm{uOR}=1.109, p=0.112 ; \mathrm{aOR}=1.257, p=0.001)$. 
Table I. Patient demographics.

\begin{tabular}{|c|c|c|c|c|}
\hline Parameter & Total, n (\%) $(\mathrm{N}=67765)$ & Staged, $\mathrm{n}(\%)(\mathrm{N}=64571)$ & Unstaged, n (\%) (N=3194) & $p$-Value* \\
\hline \multicolumn{5}{|l|}{ Age } \\
\hline$<60$ Years & $22889(33.8 \%)$ & $22059(34.2 \%)$ & $830(26.0 \%)$ & \multirow[t]{2}{*}{$<0.001$} \\
\hline$\geq 60$ Years & $44876(66.2 \%)$ & $42512(65.8 \%)$ & $2364(74.0 \%)$ & \\
\hline \multicolumn{5}{|l|}{ Gender } \\
\hline Male & $36855(54.4 \%)$ & $35164(54.5 \%)$ & $1691(52.9 \%)$ & \multirow[t]{2}{*}{0.093} \\
\hline Female & $30910(45.6 \%)$ & $29407(45.5 \%)$ & $1503(47.1 \%)$ & \\
\hline \multicolumn{5}{|l|}{ Race } \\
\hline Caucasian & $57703(85.2 \%)$ & $54968(85.1 \%)$ & $2735(85.6 \%)$ & \multirow[t]{3}{*}{0.003} \\
\hline African American & $4862(7.2 \%)$ & $4675(7.2 \%)$ & $187(5.9 \%)$ & \\
\hline Other & $5200(7.7 \%)$ & $4928(7.6 \%)$ & $272(8.5 \%)$ & \\
\hline \multicolumn{5}{|l|}{ Marital status } \\
\hline Married/living with partner & $39051(57.6 \%)$ & $37323(57.8 \%)$ & $1728(54.1 \%)$ & \multirow[t]{3}{*}{$<0.001$} \\
\hline Single & $11138(16.4 \%)$ & $10633(16.5 \%)$ & $505(15.8 \%)$ & \\
\hline $\mathrm{S} / \mathrm{D} / \mathrm{W}$ & $17576(25.9 \%)$ & $16615(25.7 \%)$ & $961(30.1 \%)$ & \\
\hline \multicolumn{5}{|l|}{ Urbanization } \\
\hline Rural & $999(1.5 \%)$ & $946(1.5 \%)$ & $53(1.7 \%)$ & \multirow[t]{3}{*}{0.672} \\
\hline Urban & $6847(10.1 \%)$ & $6526(10.1 \%)$ & $321(10.1 \%)$ & \\
\hline Metropolitan & $59919(88.4 \%)$ & $57099(88.4 \%)$ & $2820(88.3 \%)$ & \\
\hline \multicolumn{5}{|l|}{ Income ${ }^{\#}$} \\
\hline$\leq 25,000$ & $939(1.4 \%)$ & $891(1.4 \%)$ & $48(1.5 \%)$ & \multirow[t]{3}{*}{$<0.001$} \\
\hline$>25,000-50,000$ & $42468(62.7 \%)$ & $40278(62.4 \%)$ & $2190(68.6 \%)$ & \\
\hline$>50,000$ & $24358(35.9 \%)$ & $23402(36.2 \%)$ & $956(29.9 \%)$ & \\
\hline \multicolumn{5}{|l|}{ DLBCL sequence } \\
\hline First or only primary & $55777(82.3 \%)$ & $53258(82.5 \%)$ & $2519(78.9 \%)$ & \multirow[t]{2}{*}{$<0.001$} \\
\hline Not first primary & $11988(17.7 \%)$ & $11313(17.5 \%)$ & $675(21.1 \%)$ & \\
\hline \multicolumn{5}{|l|}{ Site of primary } \\
\hline Lymph nodes & $42241(62.3 \%)$ & $40090(62.1 \%)$ & $2151(67.3 \%)$ & \multirow[t]{3}{*}{$<0.001$} \\
\hline Extralymphatic & $25322(37.4 \%)$ & $24330(37.7 \%)$ & $992(31.1 \%)$ & \\
\hline Unknown & $202(0.3 \%)$ & $151(0.2 \%)$ & $51(1.6 \%)$ & \\
\hline
\end{tabular}

S/D/W: Separated/divorced/widowed. *Chi-square test. "Median annual household income.

Compared to the married population, disease in all other individuals was more likely to be unstaged $(\mathrm{uOR}=1.026$, $p=0.662 ; \mathrm{aOR}=1.208, p=0.001$ and $\mathrm{uOR}=1.249, p<0.001$; $\mathrm{aOR}=1.185, p<0.001)$. There were no differences in unstaged cases based on specific areas of residence, but after adjusting for other variables, patients with annual household incomes of $>\$ 50.000$ were less likely to have unstaged disease compared to those with household incomes of $<\$ 25,000$ (aOR=0.673, $p=0.017)$. Similarly, patients with primary cancer discovered prior to the diagnosis of DLBCL, those with extra-lymphatic sites as primary malignancy, and individuals with unknown primary sites, were more likely to be unstaged.

Survival among patients with unstaged DLBCL was greater when compared to those with distant disease (3-year RS of $59.4 \pm 1.2 \%$ vs. $52.1 \pm 0.4 \% ; p<0.0001$ and 5 -year RS of $54.3 \pm 1.4 \%$ vs. $48.0 \pm 0.4 \%$; $p<0.0001$ ), but was inferior when compared to individuals with localized and regional disease (3-year RS of $59.4 \pm 1.2 \%$ vs. $73.0 \pm 0.4 \%$ and $70.9 \pm 0.5 \%$ with $p<0.0001$ and $p<0.0001$, respectively, 5- year RS of $54.3 \pm 1.4 \%$ vs. $69.4 \pm 0.5 \%$ and $67.7 \pm 0.6 \%$ with $p<0.0001$ and $p<0.0001$, respectively) (Tables II and III).

\section{Discussion}

DLBCL is the most common type of NHL, comprising approximately $30-35 \%$ of all cases. Staging cancer is an important prognostic tool, which helps determine the appropriate course of treatment. Each year a proportion of solid tumor malignancies are unstaged (4). Our study revealed that $4.7 \%$ of the study population with DLBCL were unstaged. In addition, this cohort of patients had an inferior survival rate compared to those with localized or regional disease. These results are similar to those of Merrill et al., who reported inferior survival in patients with unstaged solid tumor malignancies (4). Gurney et al. reviewed the New Zealand Cancer Registry to determine the prevalence and prognosis of different unstaged cancer types (7). They reported that patients with unstaged tumors were significantly less likely to receive definitive surgery. Patients 
Table II. Comparison of 3-year relative survival (RS) by stage.

\begin{tabular}{|c|c|c|c|c|c|c|c|}
\hline \multirow[t]{2}{*}{ Parameter } & \multicolumn{4}{|c|}{ Overall 3-year RS + standard error (\%) } & \multicolumn{3}{|c|}{$p$-Value: Unstaged $v s$} \\
\hline & Localized & Regional & Distant & Unstaged & Localized & Regional & Distant \\
\hline All & $73.0 \pm 0.4$ & $70.9 \pm 0.5$ & $52.1 \pm 0.4$ & $59.4 \pm 1.2$ & $<0.0001$ & $<0.0001$ & $<0.0001$ \\
\hline$<60$ Years & $83.0 \pm 0.5$ & $82.5 \pm 0.6$ & $61.8 \pm 0.6$ & $76.0 \pm 1.7$ & $<0.0001$ & $<0.0001$ & $<0.0001$ \\
\hline$\geq 60$ Years & $66.4 \pm 0.6$ & $62.7 \pm 0.8$ & $46.2 \pm 0.5$ & $52.2 \pm 1.5$ & $<0.0001$ & $<0.0001$ & 0.0011 \\
\hline Male & $73.5 \pm 0.6$ & $71.0 \pm 0.8$ & $52.5 \pm 0.5$ & $58.0 \pm 1.7$ & $<0.0001$ & $<0.0001$ & $<0.0001$ \\
\hline Female & $72.4 \pm 0.7$ & $78.8 \pm 0.6$ & $63.5 \pm 0.5$ & $65.7 \pm 1.5$ & $<0.0001$ & $<0.0001$ & 0.0328 \\
\hline Caucasian & $73.6 \pm 0.5$ & $66.9 \pm 1.9$ & $53.1 \pm 0.4$ & $59.3 \pm 1.3$ & $<0.0001$ & $<0.0001$ & 0.0002 \\
\hline AA & $67.6 \pm 1.7$ & $66.9 \pm 1.9$ & $47.5 \pm 1.2$ & $57.4 \pm 4.5$ & 0.0206 & 0.0178 & 0.0592 \\
\hline Other & $70.6 \pm 1.5$ & $71.7 \pm 1.7$ & $47.0 \pm 1.3$ & $61.4 \pm 3.7$ & 0.0119 & 0.0020 & 0.0010 \\
\hline
\end{tabular}

AA: African American.

Table III. Comparison of 5 year relative survival by stages.

\begin{tabular}{|c|c|c|c|c|c|c|c|}
\hline \multirow[t]{2}{*}{ Parameter } & \multicolumn{4}{|c|}{ Overall 5-year RS \pm standard error (\%) } & \multicolumn{3}{|c|}{$p$-Value: Unstaged $v s$} \\
\hline & Localized & Regional & Distant & Unstaged & Localized & Regional & Distant \\
\hline All & $69.4 \pm 0.5$ & $67.7 \pm 0.6$ & $48.0 \pm 0.4$ & $54.3 \pm 1.4$ & $<0.0001$ & $<0.0001$ & $<0.0001$ \\
\hline$<60$ Years & $80.2 \pm 0.6$ & $80.4 \pm 0.7$ & $58.3 \pm 0.6$ & $69.5 \pm 2.1$ & $<0.0001$ & $<0.0001$ & $<0.0001$ \\
\hline$\geq 60$ Years & $62.0 \pm 0.8$ & $58.5 \pm 0.9$ & $41.5 \pm 0.6$ & $47.7 \pm 1.7$ & $<0.0001$ & $<0.0001$ & 0.0003 \\
\hline Male & $69.5 \pm 0.7$ & $67.6 \pm 0.9$ & $47.5 \pm 0.6$ & $55.3 \pm 1.9$ & $<0.0001$ & $<0.0001$ & $<0.0001$ \\
\hline Female & $69.3 \pm 0.8$ & $67.8 \pm 0.9$ & $48.6 \pm 0.6$ & $53.2 \pm 2.0$ & $<0.0001$ & $<0.0001$ & 0.0354 \\
\hline Caucasian & $69.4 \pm 0.5$ & $68.0 \pm 0.7$ & $49.1 \pm 0.5$ & $54.4 \pm 1.5$ & $<0.0001$ & $<0.0001$ & 0.0006 \\
\hline AA & $65.3 \pm 2.0$ & $63.6 \pm 2.2$ & $41.8 \pm 1.4$ & $51.1 \pm 5.2$ & 0.0092 & 0.0290 & 0.0856 \\
\hline Other & $66.5 \pm 1.8$ & $68.1 \pm 2.1$ & $41.9 \pm 1.5$ & $54.1 \pm 4.5$ & 0.0049 & 0.0008 & 0.0046 \\
\hline
\end{tabular}

AA: African American.

with unstaged DLBCL may also be less likely to receive aggressive treatment, which may explain the inferior survival outcomes in this population.

We also recorded a higher rate of unstaged disease in the elderly, similar to the results from other studies $(4,5)$. In a study conducted by Worthington et al., unstaged colon cancer was more likely in older individuals. They postulated that higher rates of unstaged cancer may be seen in the elderly due to a higher number of co-morbid conditions (5). Similarly, Yancik et al. reported a higher rate of unstaged breast cancer in elderly patients with co-morbid conditions (8). They suggest that patients with competing co-morbid conditions may be unable to tolerate certain procedures, such as axillary lymph node dissection, due to a compromised health status. In addition, Koroukian et al. reported that elderly patients may be less likely to consent to diagnostic work-up (9). Our findings suggest similar trends in elderly patients with DLBCL.

There were no differences in unstaged DLBCL based on gender, contrary to studies that reported a higher proportion of unstaged cancer among females (4). Disease in unmarried individuals was more likely to be unstaged compared to married patients, which is in concordance with other reports $(4,10,11)$. In a study conducted by Goodwin et al., married patients were noted to have superior support systems and socioeconomic stability, resulting in improved staging and treatment outcomes (11). In our study, patients with an annual income of $\$ 50,000$ or higher were less likely to have unstaged disease compared to the those with a lower annual income. Bradley et al. conducted a study to determine the effects of cancer on nursing home patients and found that Medicaid patients were more likely to have unstaged disease (12). Socioeconomic status may therefore play a significant role in DLBCL staging and treatment, as patients with low annual income may have poor access to insurance and healthcare (12-14).

We found a lower rate of unstaged DLBCL in African Americans compared to Caucasians, in contrast to the Merrill et al. study, which reported a higher rate of unstaged cancer of prostate, stomach, colon, rectum, kidney, thyroid, breast, cervix, and ovary in Caucasians (4). In addition, Lathan et al. reported higher rates of unstaged non-small cell lung cancer in 
African American patients (15), similar to the results from the Merrill et al. study. Such racial disparities have been attributed to several factors, including access to care, poor primary care, and perhaps some elemental mistrust of the medical system. More studies are needed to determine why fewer cases of unstaged DLBCL exists in the African American population.

There are several strengths and limitations of our study that warrant consideration. The SEER database provides us with detailed information on patient demographics and disease stage. The data are derived from both urban as well as rural regions, encompassing approximately $27.8 \%$ of the US population, adding to the validity of our study. However, disease-specific characteristics, including dose of radiation, number of cycles of treatment received, receipt of chemotherapy or immunotherapy, treatment complications, prognostic features such as lactate dehydrogenase level, performance status, or unfavorable cytogenetics, are not available through the SEER database. In addition, the SEER database does not provide information on co-morbid conditions, which may confound the prognosis of unstaged DLBCL.

\section{Conclusion}

In this population-based study, $4.71 \%$ of patients with DLBCL had unstaged disease. Older age, single/widowed/ divorced marital status, and lower socioeconomic status, were associated with higher rates of unstaged cases. Unstaged DLBCL was associated with inferior survival compared to localized and regional disease.

\section{Funding}

None.

\section{References}

1 Howlader N, Noone AM and Krapcho M: SEER Cancer Statistics Review, 1975-2011, National Cancer Institute. Bethesda, MD, Available online: http://seer.cancer.gov/csr/ 1975_2011/, based on November 2013 SEER data submission, posted to the SEER web site, April 2014.

2 López-Guillermo A, Colomo L, Jiménez M, Bosch F, Villamor N, Arenillas L, Muntañola A, Montoto S, Giné E, Colomer D, Beà S, Campo E and Montserrat E: Diffuse Large B-Cell Lymphoma: Clinical and Biological Characterization and Outcome According to the Nodal or Extranodal Primary Origin. J Clin Oncol 23(12): 2797-2804, 2005.
3 Armitage J: My treatment approach to patients with diffuse large B-cell lymphoma. Mayo Clinic Proc 87(2): 161-171, 2012.

4 Merrill RM, Sloan A, Anderson AE and Ryker K: Unstaged cancer in the United States: a population-based study. BMC Cancer 11(1): 402, 2011.

5 Worthington J, Koroukian S and Cooper G: Examining the characteristics of unstaged colon and rectal cancer cases. Cancer Detect Prev 32(3): 251-258, 2008.

6 National Cancer Institute. Overview of the Surveillance, Epidemiology, and End Results (SEER) Program. Available from URL: http://seer.cancer.gov/about/overview.html.

7 Gurney J, Sarfati D, Stanley J, Dennett E, Johnson C, Koea J, Simpson A and Studd R: Unstaged cancer in a population-based registry: prevalence, predictors and patient prognosis. Cancer Epidemiol 37(4): 498-504, 2013.

8 Yancik R, Wesley M and Ries L: Effect of age and comorbidity in postmenopausal breast cancer patients aged 55 years and older. JAMA 285(7): 885-892, 2001

9 Koroukian S, Xu F and Beaird H: Complexity of care needs and unstaged cancer in elders: a population-based study. Cancer Detect Prev 31(3): 199-206, 2007.

10 Van Jaarsveld C, Miles A, Edwards R and Wardle J: Marriage and cancer prevention: does marital status and inviting both spouses together influence colorectal cancer screening participation? J MedScreen 13(4): 172-176, 2006.

11 Goodwin J, Hunt W, Key C and Samet J: The effect of marital status on stage, treatment, and survival of cancer patients. JAMA 258(21): 3125-3130, 1987.

12 Bradley $\mathrm{C}$ and Lin C: Absence of cancer diagnosis and treatment in elderly Medicaid-insured nursing home residents. J Natl Cancer Inst 100(1): 21-31, 2008.

13 Roetzheim R, Pal N and Tennant C: Effects of health insurance and race on early detection of cancer. J Natl Cancer Inst 91(16): 1409-1415, 1999.

14 Miller B, Hankey B and Thomas T: Impact of sociodemographic factors, hormone receptor status, and tumor grade on ethnic differences in tumor stage and size for breast cancer in US Women. Am J Epidemiol 155(6): 534-554, 2001.

15 Lathan C, Neville B and Earle C: The effect of race on invasive staging and surgery in non-small-cell lung cancer. J Clin Oncol 24(3): 413-418, 2006.
Received February 27, 2017

Revised March 19, 2017

Accepted March 20, 2017 